\title{
Pessoas com dor e necessidades de intervenção: revisão sistemática da literatura
}

\author{
People with pain and intervention needs: systematic literature review \\ Personas con dolor y necesidades de intervención: revisión sistemática de la literatura
}

\begin{abstract}
João César Fonseca', Manuel José Lopes", Ana Filipa Ramos'
' Centro Hospitalar Lisboa Norte, Unidade de Investigação e Desenvolvimento em Enfermagem. Lisboa, Portugal. "Universidade de Évora, Escola Superior de Enfermagem São João de Deus. Évora, Portugal.
\end{abstract}

Submissão: 10-06-2011 Aprovação: 22-07-2013

\section{RESUMO}

Objectivou-se determinar qual o impacto económico e social e as necessidades de intervenção por parte dos serviços de saúde, relativamente às pessoas com dor. Foi efectuada uma pesquisa na EBSCO (CINAHL, MEDLINE, British Nursing Index), utilizando-se o método de $\mathrm{PI}[\mathrm{C}] \mathrm{O}$ e seleccionados 19 artigos do total de 325 encontrados. A dor de tipo neuropática e a com localização músculo-esquelética foram identificadas como as maiores responsáveis pelos gastos em saúde. Como factores positivamente associados à relação custo-eficácia, encontrou-se: associação do regime terapêutico com estratégias não farmacológicas, intervenção de equipas multidisciplinares especializadas no controlo da dor, continuidade de cuidados na comunidade e uma linha telefónica de apoio permanente. Conclui-se que a dor causa significativa deterioração na qualidade de vida, com incalculável impacto no desempenho das actividades de vida diária.

Descritores: Dor; Impacto Social; Económico e Intervenções do Serviço de Saúde.

\section{ABSTRACT}

The study aimed to determine the economic and social impact and that needs of intervention by the health services for persons with pain. It was conducted a search in EBSCO (CINAHL, MEDLINE, British Nursing Index), using the method of PI[C]O and selecting 19 articles of the total of 325 found. The neuropathic pain and the one with musculoskeletal location were identified as the most responsible for health expenditures. The factors positively associated with cost-effective met in the research were: combination of therapeutic regimen with non-pharmacological strategies, involvement of multidisciplinary teams specialized in pain management, continuity of care program in community and a telephone line for permanent support. It was concluded that the pain causes significant deterioration in quality of life, with incalculable impact on the performance of the activities of daily living.

Key words: Pain; Social and Economic Impact and Interventions of Heath Service.

\section{RESUMEN}

Objetivó-se determinar el impacto social y económico y las necesidades de intervención de los servicios de salud para las personas con dolor. Se realizó una búsqueda en EBSCO (CINAHL, MEDLINE, British Nursing Index), utilizando el método $\mathrm{PI}[\mathrm{C}] \mathrm{O}$, siendo seleccionados 19 artículos del total de 325 que se encontró. El tipo de dolor neuropática y con ubicación musculoesquelética son los más responsables por los gastos de salud. Como factores asociados positivamente con el costoeficacia, encontró-se: asociación de estrategias no farmacológicas con el régimen terapéutico, la intervención de equipos multidisciplinarios especializados en el tratamiento del dolor, la continuidad de la atención en la comunidad y una línea telefónica permanente. Es posible concluir que el dolor provoca un deterioro significativo en la calidad de vida, con un impacto incalculable en la ejecución de las actividades de la vida diaria.

Palabras clave: Dolor; Impacto Social; Económico y las Intervenciones de Servicios de Salud. 


\section{INTRODUÇÃO}

A dor é uma experiência que se inscreve, desde os primórdios, na história da humanidade, e tem sido, ao longo de milhares de anos, subvalorizada em prol de outros sintomas, continuando no cerne das problemáticas actuais, em paralelo com os significativos avanços científicos e tecnológicos no domínio da saúde ${ }^{(1-5)}$.

A experiência de dor é transversal a todas as etapas do ciclo de vida, associada às mais diversificadas situações fisiopatológicas vivenciadas no continuum saúde/doença por cada indivíduo(6). A dor pode ser definida como uma experiência sensorial desagradável associada a uma lesão efectiva ou potencial dos tecidos, ou descrita em termos de tal lesão ${ }^{(2-3)}$. Assim, é um dos sintomas mais comum que conduz as pessoas a procurar e utilizar os cuidados de saúde, sendo, portanto, considerada como um dos principais problemas de saúde pública ${ }^{(1-3,7)}$.

Esta revisão sistemática da literatura teve como objectivos: 1) identificar as repercussões financeiras e sociais provocadas pela situação de dor; 2) conhecer quais as intervenções adequadas, por parte dos serviços de saúde, para aumentar os ganhos em saúde, na pessoa com dor.

\section{METODOLOGIA}

Como ponto de partida para a revisão sistemática de literatura foi formulada a pergunta de investigação em formato $\mathrm{PI}[\mathrm{C}] \mathrm{O}$ (População, Intervenção, Comparação e Outcome) $)^{(8)}$ : Em relação às pessoas com dor (Population) qual o impacto económico e social (Outcome) e quais as necessidades de intervenção por parte dos serviços de saúde (Intervention)? Base de dados electrónica observada: EBSCO (CINAHL Plus with Full Text, MEDLINE with Full Text, British Nursing Index). Foram utilizadas palavras-chave com a seguinte orientação: ["Suffering" OR "Ache" OR "Pain"; "Hospital Costs" OR "Treatment Costs" OR "Medical Care Costs" OR "Health Care Costs" OR "Health Resource Utilization" e ("Suffering" OR "Ache" OR "Pain") AND ("Hospital Costs" OR "Treatment Costs" OR "Medical Care Costs" OR "Health Care Costs" OR "Health Resource Utilization")], as palavras foram procuradas em texto integral (Julho/2009), retrospectivamente até 2004, resultando em 325 artigos no total.

Como critérios de inclusão privilegiaram-se os artigos com foco na problemática da pessoa com dor, com recurso a metodologia qualitativa e/ou quantitativa ou revisão sistemática da literatura, que clarificassem o impacto social e/ou financeiro da dor e as acções implementadas pelos serviços de saúde, como forma de reduzir esse mesmo impacto. Nos critérios de exclusão inseriram-se todos os artigos com metodologia pouco clara, repetidos nas duas bases de dados, com data anterior a 2004 e todos aqueles sem correlação com o objecto de estudo. O percurso metodológico levado a cabo encontra-se exemplificado na figura 1.

Todos os artigos foram analisados por dois autores e, quando necessário, confirmados por um terceiro. Dos 325 artigos
Figura 1 - Processo de pesquisa e selecção.

che" OR "Pain"* $\mathrm{n}=55352$

- "Hospital Costs" OR "Treatment Costs" OR "Medical Care Costs" OR "Health Care Costs" OR "Health $\operatorname{ion}^{\prime * *} \mathrm{n}=9170$ MEDLINE $35744^{*}+3661 * *$ CINAHL $19608 *+5509 * *$ Filtração cronológica (Maio 2010) 2004/05/01 e 2009/05/31

- Boolean/Phrase

- Texto integral

logação dos artigos por níveis de evidência, Aão crítica e síntese do conhecim

finais realizou-se uma primeira análise focada no conteúdo presente no resumo, sendo incluídos todos aqueles que obedeciam aos critérios de inclusão estabelecidos. Nove artigos $(M E D L I N E=6$ e CINAHL $=13)$ resultaram desta filtração, consensual entre três autores, constituindo o material final de análise. Estes artigos foram agrupados por níveis de evidência, com a finalidade de identificar as diferentes metodologias utilizadas em cada um deles, bem como a amostra e as técnicas de recolha de dados que se referiam. Consequentemente, realizou-se uma apreciação crítica do seu conhecimento e síntese, com o objectivo de aumentar o conhecimento sobre o fenómeno da dor.

\section{RESULTADOS}

Para tornar perceptível e transparente a metodologia utilizada, explicita-se a listagem dos 19 artigos (Quadro 1), que constituíram o substrato para a elaboração da discussão e respectivas conclusões. Para avaliarmos os níveis de evidência, utilizámos seis níveis de evidência: Nível I - revisões sistemáticas (meta análises/ linhas de orientação para a prática clínica com base em revisões sistemáticas); Nível II - estudo experimental; Nível III - estudos quase experimentais; Nível IV - estudos não experimentais; Nível V - relatório de avaliação de programa/ revisões de literatura; Nível VI - opiniões de autoridades / painéis de consenso (8).

\section{DISCUSSÃO}

Como resultado da análise dos artigos supra-referenciados é possível denotar que, quanto ao tipo de dor, a dor neuropática ocupa um lugar proeminente, sendo destacada em cinco dos dezanove artigos ${ }^{(1-2,5,12,20)}$. O consumo em recursos de saúde é três vezes mais elevado aquando a presença de dor neuropática, fortemente correlacionado com a predisposição para desenvolverem outras co-morbilidades, como a fibromialgia, osteo-artrite, doença coronária e depressão ${ }^{(2)}$. Mais de metade das pessoas com dor crónica não estavam satisfeitas com o seu plano de terapêutica, apesar da elevada adesão ao regime 
Quadro 1 - Descrição dos resultados.

\begin{tabular}{|c|c|c|}
\hline Estudo & Intervenções & Resultados \\
\hline $\begin{array}{l}\text { Berger, Dukes e Oster (2004) } \\
\text { - } \quad \mathrm{n}=55,686 \text { pessoas com } \\
\text { dor neuropática } \\
\text { - Nível de Evidência II }\end{array}$ & $\begin{array}{l}\text { Análise de base de dados de seguros } \\
\text { de saúde nos EUA, em } 2000 \text {, de todas } \\
\text { as pessoas com } 18 \text { anos ou mais, } \\
\text { com duas ou mais consulta por dor } \\
\text { neuropática }\end{array}$ & $\begin{array}{l}\text { Resultados sugerem que as pessoas com dor } \\
\text { neuropática estão em condições de saúde menos } \\
\text { favoráveis e têm gastos em saúde mais elevados }\end{array}$ \\
\hline $\begin{array}{l}\text { Breen et al. (2005) }{ }^{(9)} \\
\text { - Nível de Evidência II } \\
\text { - } \mathrm{n}=87 \text { enfermeiras e } \\
\text { doentes }\end{array}$ & $\begin{array}{l}\text { Utilização de focus grupo e análise de } \\
\text { conteúdo }\end{array}$ & $\begin{array}{l}\text { Resultados relatam a capacidade inadequada para } \\
\text { lidar com as necessidades multidimensionais } \\
\text { da pessoa com dor, o que pode permitir a sua } \\
\text { progressão para crónica e inflaciona os custos em } \\
\text { saúde }\end{array}$ \\
\hline $\begin{array}{l}\text { Ekman, Johnell e Lidgren } \\
\text { (2005) } \\
\text { • Nível de Evidência III }\end{array}$ & $\begin{array}{l}\text { Utilização de metodologia } \\
\text { quantitativa na relação custos em } \\
\text { serviços de saúde e absentismo } \\
\text { laboral }\end{array}$ & $\begin{array}{l}\text { O custo total com a dor na região dorsal posterior } \\
\text { foi de } 1860 \text { milhões de EUR na Suécia em } 2001 \text {. } \\
\text { Os gastos indirectos, com a perda de produtividade } \\
\text { foram } 84 \% \text { do custo total }\end{array}$ \\
\hline $\begin{array}{l}\text { Ahles et al. (2006) } \\
\text { - Nível de Evidência III } \\
\text { - } \mathrm{n}=644 \text { pessoas com dor / } \\
693 \text { pessoas sem dor }\end{array}$ & Apoio telefónico a pessoas com dor & $\begin{array}{l}\text { Facilitou a resolução de problemas e/ ou disfunções } \\
\text { psicossociais e aumentou a eficácia no controlo da } \\
\text { dor }\end{array}$ \\
\hline $\begin{array}{l}\text { Zenker et al. (2006) }{ }^{(11)} \\
\text { - Nível de Evidência III } \\
\text { - } \mathrm{n}=163 \text { pessoas com dor } \\
\text { crónica neuropática }\end{array}$ & Utilização de metodologia qualitativa & $\begin{array}{l}\text { O sucesso terapêutico aumentou consoante } \\
\text { um maior grau de motivação, visível na menor } \\
\text { utilização dos cuidados de saúde e recurso a } \\
\text { analgésicos opióides }\end{array}$ \\
\hline $\begin{array}{l}\text { Marchettini, Dukes e Ryan } \\
\text { (2006) } \\
\text { - Nível de Evidência III } \\
\text { - } \mathrm{n}=81 \text { doentes }\end{array}$ & $\begin{array}{l}\text { Estudo transversal em } 81 \text { doentes com } \\
\text { dor neuropática cervical, em } 6 \text { países } \\
\text { europeus }\end{array}$ & $\begin{array}{l}\text { A dor foi relatada com intensidade moderada ou } \\
\text { severa em } 89 \% \text { dos participantes, apesar de } 94 \% \\
\text { estarem medicados. A interrupção laboral ocorreu } \\
\text { em } 62 \% \text { dos mesmos. A presença permanente } \\
\text { de dor interferiu negativamente na capacidade } \\
\text { funcional e percepção sobre o estado de saúde }\end{array}$ \\
\hline $\begin{array}{l}\text { Forberg et al. (2006) }{ }^{(3)} \\
\text { - Nível de Evidência I } \\
\text { - } \mathrm{n}=\text { Análise de } 1,000 \\
\text { atendimentos }\end{array}$ & $\begin{array}{l}\text { Análise de 1,000 atendimentos } \\
\text { consecutivos no Serviço de Urgência, } \\
\text { com dor pré-cordial. Os custos do } \\
\text { atendimento foram obtidos a partir do } \\
\text { sistema de economia do hospital }\end{array}$ & $\begin{array}{l}\text { O custo médio por visita/ pessoa foi } 26.800 \\
\text { em coroa sueca, detectando-se a existência de } \\
\text { um número considerável de falsos positivos. O } \\
\text { internamento representou } 73 \% \text { dos custos totais. }\end{array}$ \\
\hline $\begin{array}{l}\text { Gatchel e Okifuji (2006) }{ }^{(13)} \\
\text { - Nível de Evidência IV } \\
\text { • } \mathrm{n}=14 \text { estudos }\end{array}$ & $\begin{array}{l}\text { Pesquisa de literatura sobre a dor } \\
\text { crónica durante a última década nas } \\
\text { bases de dados MEDLINE e PSYCHLIT }\end{array}$ & $\begin{array}{l}\text { Estima-se que } 10 \% \text { a } 20 \% \text { da população adulta } \\
\text { sente dor persistente de duração mínima de três } \\
\text { meses. Cerca de } 62 \% \text { dos indivíduos tiveram mis } \\
\text { que um episodio dor no ano, e } 40 \% \text { relataram que } \\
\text { tem dor crónica. }\end{array}$ \\
\hline $\begin{array}{l}\text { Weiner et al. (2006) }{ }^{(14)} \\
\text { - Nível de Evidência } V \\
\text { - } \mathrm{n}=111 \text { pessoas com mais } \\
\text { de } 65 \text { anos de idade }\end{array}$ & $\begin{array}{l}\text { Estudo 1: Consulta nacional e na } \\
\text { Pensilvânia sobre o número de } \\
\text { doentes e encargos com todos } \\
\text { os pacientes, e para aqueles com } \\
\text { inespecíficos dor lombar. } \\
\text { Estudo 2: foram estudados } 111 \text { idosos } \\
\text { com dor lombar crônica }\end{array}$ & $\begin{array}{l}\text { A documentação e os estudos de diagnóstico } \\
\text { são cada vez mais benéficos. Verifica-se que o } \\
\text { tratamento seja responsável por uma proporção } \\
\text { significativa dos custos com a dor lombar. Mais } \\
\text { estudos para verificar a adequação com que os } \\
\text { procedimentos de imagem e tratamentos pouco } \\
\text { invasivos são utilizados, bem como a relação dos } \\
\text { seus efeitos sobre os resultados do paciente }\end{array}$ \\
\hline
\end{tabular}




\begin{tabular}{|c|c|c|}
\hline Estudo & Intervenções & Resultados \\
\hline $\begin{array}{l}\text { Katz (2006) } \\
\text { - Nível de Evidência III } \\
\bullet \mathrm{n}=12 \text { estudos }\end{array}$ & Revisão da literatura & $\begin{array}{l}\text { O custo total da dor lombar nos Estados Unidos } \\
\text { ultrapassam os US \$ } 100 \text { bilhões por ano. Dois } \\
\text { terços desses custos são indirectos, devido a } \\
\text { perdas salariais e redução da produtividade. Como } \\
\text { os custos indirectos dependem fortemente de } \\
\text { mudanças no status do trabalho, os custos totais são } \\
\text { difíceis de calcular. }\end{array}$ \\
\hline $\begin{array}{l}\text { Tölle, Xub, Sadosky (2006) }{ }^{(5)} \\
\text { - Nível de Evidência III } \\
\text { • } \mathrm{n}=140 \text { pessoas de seis } \\
\text { países Europeus }\end{array}$ & $\begin{array}{l}\text { Pacientes que responderam a um } \\
\text { questionário que incluía a intensidade } \\
\text { da dor e a interferência da dor no } \\
\text { quotidiano, um Inventário Breve } \\
\text { de Dor, e questões relacionadas } \\
\text { à produtividade e à utilização de } \\
\text { recursos de saúde }\end{array}$ & $\begin{array}{l}\text { Os pacientes relataram que interferência moderada, } \\
\text { apesar de } 91 \% \text { dos pacientes referem o uso de } \\
\text { fármacos para a dor. O uso de medicamentos } \\
\text { de prescrição para a ansiedade concomitante, } \\
\text { depressão ou distúrbios do sono foi referido por } \\
43 \% \text { dos pacientes. Desemprego foi apontado } \\
\text { por } 35 \% \text { dos pacientes. A intensidade da dor foi } \\
\text { significativamente associada com redução de } \\
\text { avaliação da saúde do estado, pontuação maior. A } \\
\text { dor foi a perturbação mais significativa no emprego } \\
\text { e que levou a mais visitas do médico pela maioria } \\
\text { dos pacientes. }\end{array}$ \\
\hline $\begin{array}{l}\text { Pizzi et al. (2006) }{ }^{(16)} \\
\text { - Nível de Evidência IV } \\
\text { - } \mathrm{n}=2459 \text { trabalhadores de } \\
\text { seis grandes empresas dos } \\
\text { EUA }\end{array}$ & $\begin{array}{l}\text { Os custos de saúde foram calculados } \\
\text { a partir do valor pago para os créditos } \\
\text { dos trabalhadores de escritório } \\
\text { para atendimento em ambulatório, } \\
\text { internamento e medicamentos de } \\
\text { prescrição. }\end{array}$ & $\begin{array}{l}61,5 \% \text { perdeu um dia de trabalho durante o } \\
\text { período de estudo de } 3 \text { anos. O número total de } \\
\text { dias perdidos por faltas por doença e os benefícios } \\
\text { por incapacidade a curto prazo foram de } 331 \\
242, \text { no valor de cerca de US } \$ 39.4 \text { milhões em } \\
\text { salários durante o período de } 3 \text { anos. Combinando } \\
\text { os custos médicos e de farmácia foram de US } \$ \\
4607 \text { por funcionário por ano so relacionados à } \\
\text { dor. O impacto estimado de dor crónica para cada } \\
\text { empregador, que contribuiu para o conjunto de } \\
\text { dados foi de aproximadamente US } \$ 2.1 \text { milhões } \\
\text { por ano. }\end{array}$ \\
\hline $\begin{array}{l}\text { Rodriguez e Garcia (2007) (1) } \\
\text { - Nível de Evidência V } \\
\bullet \mathrm{n}=504 \text { doentes }\end{array}$ & $\begin{array}{l}\text { Dois grupos de pacientes foram } \\
\text { constituídos segundo o tipo de gestao } \\
\text { da dor. Grupo } 1 \text { em que a gestão era } \\
\text { realizada por especialistas. Grupo } 2 \\
\text { em que a gestão era feita em clínicas } \\
\text { de dor }\end{array}$ & $\begin{array}{l}\text { A média ajustada do custo mensal total foi de } \\
€ 363 \text { por paciente, não havendo diferenças } \\
\text { estatisticamente significativas entre o tipo de } \\
\text { atendimento: } 376 €(1) \text {, contra } € 344 \text { ( } 2) \text { [p }= \\
0,626] \text {. Aquisição média mensal de despesas com } \\
\text { medicamentos foi maior no grupo } 2: € 131 \text { ( } 34,8 \% \\
\text { do custo total), contra } € 80(23,3 \%) \text { por paciente } \\
(p=0,0001) \text {. No entanto, idas à urgência, visitas } \\
\text { aos cuidados primários e o número de fármacos no } \\
\text { tratamento foi significativamente maior no grupo } 1 \text {. }\end{array}$ \\
\hline $\begin{array}{l}\text { Koo }(2007) \text { (17) } \\
\text { - Nível de Evidência } V \\
\bullet \mathrm{n}=11 \text { pessoas com dor }\end{array}$ & $\begin{array}{l}\text { Estudo com o objectivo de identificar } \\
\text { os factores de custo no tratamento } \\
\text { da dor pós-operatória e as sequelas } \\
\text { económicas subtratamento da dor são } \\
\text { revistos. }\end{array}$ & $\begin{array}{l}\text { O subtratamento da dor aguda pós-operatória tem } \\
\text { implicações importantes para os sistemas de saúde } \\
\text { do ponto de vista da economia e da satisfação do } \\
\text { paciente. O tratamento inadequado da dor pós- } \\
\text { operatória pode ter importantes consequências } \\
\text { económicas associadas a uma maior morbidade e a } \\
\text { hospitalização prolongada e readmissões. }\end{array}$ \\
\hline $\begin{array}{l}\text { Roggeri et al. (2007) }{ }^{(18)} \\
\text { - Nível de Evidência II } \\
\text { - } \mathrm{n}=935 \text { doentes }\end{array}$ & $\begin{array}{l}\text { Estudo retrospectivo epidemiológico } \\
\text { observacional, sobre a utilização de } \\
\text { opióides na Itália, tanto em termos } \\
\text { de doses como na duração do } \\
\text { tratamento. }\end{array}$ & $\begin{array}{l}\text { O custo total diário por paciente antes da primeira } \\
\text { prescrição de opióides foi } 11,36 € \text {, enquanto após } \\
\text { a primeira prescrição de opióides, aumentou para } \\
21,12 € \text {. }\end{array}$ \\
\hline
\end{tabular}




\begin{tabular}{|c|c|c|}
\hline Estudo & Intervenções & Resultados \\
\hline $\begin{array}{l}\text { Kronborg, Handberg e Axelsen } \\
(2008)^{(4)} \\
\text { - Nível de Evidência VI } \\
\text { - } \mathrm{n}=166 \text { doentes }\end{array}$ & As entrevistas estruturadas telefónicas & $\begin{array}{l}\text { Os custos de saúde devido à dor crônica são } \\
\text { elevados particular durante o primeiro ano após } \\
\text { o início da dor, e permanecem altos quando } \\
\text { comparados com os custos dos cuidados de saúde } \\
\text { antes do início da dor. A dor crónica provoca } \\
\text { perdas de produção no trabalho, bem como } \\
\text { limitação das actividades de lazer }\end{array}$ \\
\hline $\begin{array}{l}\text { Ho et al. (2008) }{ }^{(19)} \\
\text { - Nível de Evidência I } \\
\text { - } \mathrm{n}=75 \text { pais de crianças }\end{array}$ & $\begin{array}{l}\text { Entrevistas semi-estruturada a pais } \\
\text { de crianças na sua primeira visita } \\
\text { à clínica da dor. Os participantes } \\
\text { apresentavam dor há pelo menos três } \\
\text { meses, eram atendidos num serviço } \\
\text { ambulatório multidisciplinar de dor. } \\
\text { As informações foram obtidas durante } \\
\text { uma }\end{array}$ & $\begin{array}{l}\text { Os pais relataram na visita inúmeras idas aos } \\
\text { serviços de saude relacionadas com os seus filhos } \\
\text { em que a dor era a causa. Referiram altos custos } \\
\text { financeiros [por exemplo, encargos de consultas } \\
\text { ambulatoriais e tempo gasto em consultas médicas } \\
\text { e dias de trabalho perdida]. Evidências preliminares } \\
\text { sugerem que o envolvimento em um programa } \\
\text { multidisciplinar pode estar associada a menor } \\
\text { utilização dos cuidados de saúde e os encargos } \\
\text { indirectos. }\end{array}$ \\
\hline $\begin{array}{l}\text { Manca et al (2008) }{ }^{(20)} \\
\text { - Nível de Evidência III } \\
\text { - } \mathrm{n}=100 \text { patients with } 18 \\
\text { years or older }\end{array}$ & $\begin{array}{l}\text { Questionários sobre os custos e os } \\
\text { resultados foram avaliados para cada } \\
\text { paciente durante os primeiros seis } \\
\text { meses do tratameto. }\end{array}$ & $\begin{array}{l}\text { Os doentes com pé neuropático e com de dor na } \\
\text { região posterior do tronco apresentam custos mais } \\
\text { elevados para os sistemas de saúde. }\end{array}$ \\
\hline $\begin{array}{l}\text { Smeets et al. (2008) } \\
\text { - Nível de Evidência III } \\
\bullet \mathrm{n}=172 \text { patients }\end{array}$ & $\begin{array}{l}\text { O Questionário de Incapacidade } \\
\text { de Roland era usado para calcular a } \\
\text { relação custo-eficácia e a reduzir da } \\
\text { incapacidade e o ganho em anos de } \\
\text { vida ajustado pela qualidade. }\end{array}$ & $\begin{array}{l}\text { Redução da incapacidade e o ganho de anos } \\
\text { de vida não diferiram significativamente entre } \\
\text { o tratamento combinado e as modalidades de } \\
\text { tratamento único. }\end{array}$ \\
\hline
\end{tabular}

medicamentoso, o que as levou à automedicação como tentativa de mitigar a dor, aumentando consideravelmente o pobre controlo da sintomatologia, a ocorrência de efeitos adversos, bem como necessidade de hospitalização ${ }^{(1)}$.

A localização da dor na região dorsal posterior é a que assume maior relevo, de entre os artigos analisados, sendo descrita em seis dos dezanove $\operatorname{artigos}^{(9-11,14-15,20)}$.

Os custos em serviços de saúde são particularmente elevados durante o primeiro ano após o surgimento da dor e aumentam posteriormente a esse período, com consequente repercussão na perda da capacidade funcional e de produtividade $^{(4,12)}$, manifestada pela incapacidade de levar a cabo a actividade laboral, sobretudo na dor músculo-esqueléti$\mathrm{ca}^{(5,10,15-16)}$ de se auto-recrearem, estudarem, realizarem as tarefas domésticas ou tomar conta dos próprios filhos. Deste modo, o impacto da dor é manifestamente evidente nas profundas alterações ao nível das actividades de vida diárias, o que foi apontado em dezoito, dos dezanove artigos, o que resulta em custos directos e indirectos.

A experiência de (con)viver com a dor interpretada como severa esteve profundamente relacionada com a percepção de um estado de saúde classificado como "mau" ${ }^{(5,12)}$, francamente relacionado com a presença de dor constante ${ }^{(13)}$.

$\mathrm{O}$ recurso às estratégias farmacológicas assume-se como a mais importante e eficaz forma de atenuar a dor, mas que incrementam os gastos em saúde ${ }^{(18)}$, o custo diário, por pessoa com dor, após prescrição de opióides duplica. Ainda, das pessoas medicadas para o alívio da dor, quase metade utiliza, em simultâneo, fármacos para distúrbios do sono, ansiedade e depressão, representando um elevado índice de co-morbilidade, que transcende o nível físico e exerce um incalculável impacto na esfera emocional, psicológica e social, na pessoa com dor ${ }^{(5)}$. O sofrimento psicológico é visível na procura de ajuda assistencial especializada na vertente de saúde mental e psiquiátrica, pelo menos uma vez por mês ${ }^{(5,12)}$.

O recurso a terapias complementares tornou a dor mais suportável, ocupando principal destaque a acupunctura (43\%), a massagem $(42 \%)$ e a reflexologia $(32 \%)^{(4)}$. As estratégias não farmacológicas foram investigadas com o objectivo major de reduzir os gastos em saúde acoplados à dor, a relação custo-eficácia da implementação de Programas de Compreensão da Dor, centrados numa abordagem biopsicossocial, com intervenção de uma equipa multiprofissional, concluindo que pode ser mais económica para o sistema de saúde e promotora de maior qualidade de vida para as pessoas com dor ${ }^{(13)}$, em comparação com a utilização unilateral da medicina convencional $^{(21-22)}$. No que diz respeito à intervenção dos serviços de saúde no combate a dor ${ }^{(23)}$, verificou-se um maior número de idas à urgência e aos cuidados de saúde primários, bem como a utilização de um maior número de fármacos para atenuar a dor nas pessoas, cuja assistência é prestada por profissionais de saúde generalistas ${ }^{(1)}$. A desigualdade no acesso a 
Quadro2 - Impacto económico, social e necessidades de intervenção dos serviços de Saúde.

\begin{tabular}{|c|c|}
\hline $\begin{array}{c}\text { Impacto económico } \\
\text { e social }\end{array}$ & $\begin{array}{l}\text { - Dor neuropática com custos mais elevados; } \\
\text { - Dor músculo esquelética tem maior impacto na população activa; } \\
\text { - Custos mais elevados dos sistemas de saúde, com intervençõeso não estruturadas, em relação } \\
\text { - Doentes em programas de tratamento sem resolução da sintomatologia duplicam custos ao fim } \\
\text { de um ano; } \\
\text { - Reduzida satisfação com plano terapêutico conduz à automedicação e aumenta a taxa de } \\
\text { - hospitalizações; } \\
\text { - Não resolução da intensidade de dor classificada como moderada ou intensa interfere no } \\
\text { - Níveis elevados de absentismo ao trabalho; } \\
\text { - Procura especializada em cuidados de saúde mental e psiquiátrica, em mais de } 50 \% \text { das } \\
\text { pessoas com dor. }\end{array}$ \\
\hline $\begin{array}{l}\text { Intervenção dos } \\
\text { serviços de saúde }\end{array}$ & $\begin{array}{l}\text { - Implementação de programas follow-up; } \\
\text { - Gestão de casos em equipa multidisciplinar especifica; } \\
\text { - Continuidade de cuidados (consultas, intervenção na comunidade); } \\
\text { - Linha telefónica de aconselhamento 24h; } \\
\text { - Formação especifica dos profissionais de saúde na problemática da dor; } \\
\text { - Estratégias farmacológicas em associação com as estratégicas não farmacológicas. }\end{array}$ \\
\hline
\end{tabular}

profissionais de saúde especializados no controlo da dor, em que menos de metade referiram ter sido alvo de uma avaliação, pela consulta da dor ${ }^{(5)}$.

A implementação de uma efectiva continuidade de cuidados por profissionais de saúde qualificados, com a utilização de uma linha telefónica de apoio permanente se revelou crucial no desenvolvimento de mecanismos de coping e de competências de auto-gestão para intervenção activa no seu plano de saúde ${ }^{(7,19)}$. A capacidade inadequada dos profissionais de saúde para tratar e/ou cuidar das necessidades complexas e muldimensionais da pessoa com dor ${ }^{(24)}$, pode constituir um significativo factor para inflacionar os custos em saúde, ocasionado pelo deficit de intervenção atempada e adequada, que transforma frequentemente a dor aguda em crónica ${ }^{(9)}$.

Dos factores apontados responsáveis pela dilatação dos gastos em saúde, distinguem-se a medicalização dos cuidados de saúde, associada à prática de uma medicina defensiva, sugerindo uma maior exploração dos exames auxiliares de diagnóstico e meios de tratamento invasivos e não invasivos ${ }^{(25)}$, bem como quais os seus efeitos na pessoa com dor, de modo a minimizar o impacto económico de intervenções desnecessárias e os seus custos indirectos ${ }^{(3,14)}$.

Apresentamos no Quadro 2, de forma sistemática, o resumo dos principais indicadores relativos ao impacto económico e social e necessidades de intervenção dos serviços de saúde, discutidos ao longo deste artigo de revisão.

\section{CONCLUSÕES}

Relativamente ao tipo de dor, a dor neuropática emerge como aquela a que se atribui um maior custo em saúde, relacionado com a presença de dor constante de intensidade moderada. A dor músculo-esquelética, com localização na região dorsal posterior surge como a mais frequente, sobretudo em idade activa.
As profundas alterações inferidas nas actividades de vida diárias foram amplamente referenciadas, principalmente na perda significativa da capacidade de mestria, que acontece em mais de metade das pessoas com dor, que conduz ao absentismo, a incapacidade e a aposentação precoce ou desemprego. A utilização isolada dos recursos farmacológicos demonstrou-se insuficiente. As estratégias não farmacológicas com intervenção física (ex. acupunctura, massagem, reflexologia) ou psicológica (ex. psicoterapia) constituíram um importante recurso na atenuação da dor, especialmente quando combinadas com o regime medicamentoso, com potencial para abater os custos em saúde da prática de uma medicina convencional. Todavia, é possível inferir que, embora exista uma grande cobertura no tratamento farmacológico da dor, a sua repercussão no viver humano afecta francamente o bem-estar psicológico, visível na procura de cuidados de saúde na vertente de saúde mental e psiquiátrica, pelo menos uma vez por mês.

$\mathrm{Na}$ intervenção e no apoio assistencial dos serviços de saúde prestados à pessoa com dor destacam-se vários factores que interferem positivamente na economia de saúde: o acompanhamento por profissionais de saúde especializados no controlo da dor com implementação efectiva de programas follow-up, a presença de uma equipa multidisciplinar, a continuidade de cuidados e a existência de uma linha telefónica de apoio permanente, decorrentes das necessidades individuais, complexas e multidimensionais ocasionadas pela dor. Os gastos em saúde com a dor disparam um ano após o seu surgimento. Neste sentido, o impacto da dor transcende em larga escala a economia de saúde, afectando fortemente a economia global, em que, por exemplo, dois terços dos custos totais estão estreitamente associados à perda da capacidade de produtividade, resultando em custos incalculáveis para a pessoa/família/sociedade, pelo que se sugere uma abordagem integrada da presente problemática. 


\section{REFERÊNCIAS}

1. Rodríguez MJ, García AJ. A Registry of the aetiology and costs of neuropathic pain in pain clinics: results of the registry of aetiologies and costs (REC) in neuropathic Pain Disorders Study. Clin Drug Investig [periódico na internet]. 2007 Nov [acesso em 13 dez 2012]; 27(11):771-782. Disponível em: http://www.ncbi.nlm.nih.gov/pubmed/17914896

2. Berger A, Dukes EM, Oster G. Clinical characteristics and economic costs of patients with painful neuropathic disorders. The Journal Of Pain [periódico na internet]. 2004 Abr [acesso em 13 dez 2013];5(3):143-149. Disponível em: http://www.jpain.org/article/S1526-5900(03)00924-6/ abstract

3. Forberg JL, Henriksen LS, Edenbrandt L, Ekelund U. Direct hospital costs of chest pain patients attending the emergency department: a retrospective study. BMC Emerg Med [periódico na internet]. 2006 [acesso em 13 dez 2012];66. Disponível em: http://www.ncbi.nlm.nih. gov/pubmed/16674827

4. Kronborg C, Handberg G, Axelsen F. Health care costs, work productivity and activity impairment in non-malignant chronic pain patients. Eur J Health Econ [periódico na internet]. 2009 Fev [acesso em 13 dez 2012];10(1):5-13. Disponível em: http://www.ncbi.nlm.nih.gov/pubmed/18256865

5. Tölle T, Xu X, Sadosky A. Painful diabetic neuropathy: a cross-sectional survey of health state impairment and treatment patterns. J Diabetes its Complicat [periódico na internet]. 2006 Jan [acesso em 13 dez 2012];20(1):26-33. Disponível em: http://www.ncbi.nlm.nih.gov/pubmed/16389164

6. Henzler T, Gruettner J, Meyer M, Rothhaar B, Apfaltrer P, Metzger $\mathrm{F}$, et al. Coronary computed tomography and triple rule out $\mathrm{CT}$ in patients with acute chest pain and an intermediate cardiac risk for acute coronary syndrome: part 2: economic aspects. Eur J Radiol [periódico na internet]. 2013 Jan [acesso em 13 jul 2012];82(1):106-111. Disponível em: http://www.ncbi.nlm.nih.gov/pubmed/22835878

7. Ahles TA, Wasson JH, Seville JL, Johnson DJ, Cole BF, McKinstry $\mathrm{E}$, et al. A controlled trial of methods for managing pain in primary care patients with or without co-occurring psychosocial problems. Ann Fam Med [periódico na internet]. 2006 Jul [acesso em 13 dez 2012];4(4):341-350. Disponível em: http://www.ncbi.nlm.nih.gov/pubmed/16868238.

8. Melnyk BM, Fineout-Overholt E. Evidence-based practice in nursing \& healthcare: a guide to best practice. Philadelphia: Lippincott Williams \& Wilkins; 2005.

9. Breen A, Carr E, Mann E, Crossen-White H. Acute back pain management in primary care: a qualitative pilot study of the feasibility of a nurse-led service in general practice. J Nurs Manag [periódico na internet]. 2004 Maio [acesso em 13 dez 2012];12(3):201-209. Disponível em: http://www.ncbi.nlm.nih.gov/pubmed/15089958

10. Ekman M, Johnell O, Lidgren L. The economic cost of low back pain in Sweden in 2001. Acta Orthop [periódico na internet]. 2005 Abr [acesso em 13 dez 2012];76(2):275-284. Disponível em: http://www.ncbi.nlm.nih.gov/pubmed/ 16097556
11. Zenker S, Petraschka M, Schenk M, Reißhauer A, Newie $\mathrm{T}$, Spies $\mathrm{C}$, et al. Adjustment to chronic pain in back pain patients classified according to the motivational stages of chronic pain management. J Pain [periódico na internet]. 2006 Jun [acesso em 13 dez 2012];7(6):417-427. Disponível em: http://www.ncbi.nlm.nih.gov/pubmed/16750798

12. Marchettini $P$, Dukes EM, Ryan KL. Burden and treatment patterns of neuropathic pain in patients with cervical radiculopathy. J Neuropathic Pain Symptom Palliation [periódico na internet]. 2006 Jun [acesso em $13 \mathrm{dez}$ 2012];2(2):43-51. Disponível em: http://informahealthcare.com/doi/abs/10.3109/J426v02n02_04

13. Gatchel R, Okifuji A. Evidence-based scientific data documenting the treatment and cost-effectiveness of comprehensive pain programs for chronic nonmalignant pain. J Pain [periódico na internet]. 2006 Nov [acesso em $13 \mathrm{dez}$ 2012];7(11):779-793. Disponível em: http://www.ncbi. nlm.nih.gov/pubmed/17074616

14. Weiner DK, Kim YS, Bonino P, Wang T. Low back pain in older adults: are we utilizing healthcare resources wisely? Pain Med [periódico na internet]. 2006 Abr [acesso em 13 dez 2012];7(2): 143-150. Disponível em: http://www. ncbi.nlm.nih.gov/pubmed/16634727.

15. Katz J. Lumbar disc disorders and low-back pain: socioeconomic factors and consequences. J Bone Joint Surg Am [periódico na internet]. 2006 Abr [acesso em $13 \mathrm{dez}$ 2012];88 Suppl 2:21-24. Disponível em: http://www. ncbi.nlm.nih.gov/pubmed/16595438

16. Pizzi LT, Carter CT, Howell JB, Vallow SM, Crawford AG, Frank ED. Work loss, healthcare utilization, and costs among US employees with chronic pain. Dis Manage Health Outcomes [periódico na internet]. 2005 Jun [acesso em 13 dez 2012];13(3):201-208. Disponível em: http://link.springer.com/content/pdf/10.2165\% 2F00115677-200513030-00005.pdf\#page-1

17. Koo PJ. Addressing stakeholders' needs: economics and patient satisfaction. American J Health-System Pharmacy: AJHP [periódico na internet]. 2007 Mar [acesso em 13 dez 2012];64:(6 Suppl 4):S11-S15. Disponível em: http://www.unboundmedicine.com/medline/citation/17353555/Addressing_stakeholders'_needs:_economics_and_patient_satisfaction_

18. Roggeri D, Saramin C, Terrazzani G, Zusso M, Giusti P, Chinellato A. Resource consumption and costs of treating pain in patients affected by cancer in a district of northeast Italy. J Italian Pharmacological Society [periódico na internet]. 2007 Out [acesso em 13 dez 2012];56(4):329-334. Disponível em: http://www.unboundmedicine.com/medline/citation/17851088/Resource_consumption_and_ costs_of_treating_pain_in_patients_affected_by_cancer_ in_a_district_of_northeast_Italy_

19. Ho IK, Goldschneider KR, Kashikar-Zuck S, Kotagal U, Tessman C, Jones B. Healthcare utilization and indirect burden among families of pediatric patients with chronic pain. J Musculoskeletal Pain [periódico na 
internet]. 2008 Jun [acesso em 13 dez 2012];16(3):155164. Disponível em: http://informahealthcare.com/doi/ abs/10.1080/10582450802161853

20. Manca A, Kumar K, Taylor RS, Jacques L, Eldabe S, Meglio $M$, et al. Quality of life, resource consumption and costs of spinal cord stimulation versus conventional medical management in neuropathic pain patients with failed back surgery syndrome (PROCESS trial). Eur J Pain [periódico na internet]. 2008 Nov [acesso em $13 \mathrm{dez}$ 2012];12(8):1047-1058. Disponível em: http://www.ncbi. nlm.nih.gov/pubmed/18359255

21. Smeets RJ, Severens JL, Beelen S, Vlaeyen JW, Knottnerus JA. More is not always better: cost-effectiveness analysis of combined, single behavioral and single physical rehabilitation programs for chronic low back pain. Eur J Pain [periódico na internet]. 2009 [acesso em $13 \mathrm{dez}$ 2013];13(1):71-81. Disponível em: http://www.ncbi.nlm. nih.gov/pubmed/18434221
22. Larner D. Chronic Pain Transition: A Concept Analysis. Pain Manag Nurs [periódico na internet]. 2013 Jun [acesso em 7 jul 2013]. Disponível em: http://www.ncbi.nlm. nih.gov/pubmed/23769800.

23. Wyshak G. Health disparities paradoxes: depression and chronic back pain in college alumnae and U.S. women, 39-49. Scand J Public Health [periódico na internet]. 2013 Maio [acesso em 7 jul 2013];41(3):230-232. Disponível em: http://www.ncbi.nlm.nih.gov/pubmed/23287395

24. Wenham CY, Conaghan PG. New horizons in osteoarthritis. Age Ageing [periódico na internet]. 2013 Maio [acesso em 7 jul 2013];42(3): 272-278. Disponível em: http:// www.ncbi.nlm.nih.gov/pubmed/23568255

25. Nuckols TK, Escarce JJ, Asch SM. The effects of quality of care on costs: a conceptual framework. Milbank Q [periódico na internet]. 2013 Jun [acesso em 7 jul 2013];91(2):316-353. Disponível em: http://www.ncbi. nlm.nih.gov/pubmed/23758513 